\title{
Increasing Mild Enterovirus Cases Provides An Important Signal of Up-coming Trends in Elevating Severe Enterovirus Cases
}

\author{
Ta-Chien Chan*1, Rung-Hung Chen² and Chwan-Chuen King ${ }^{3}$ \\ ${ }^{1}$ Center for GIS, Research Center for Humanities and Social Sciences, Academia Sinica, Taipei City, Taiwan; ${ }^{2}$ Department of \\ Medicine, College of Medicine, National Taiwan University, Taipei City, Taiwan; ${ }^{3}$ nstitute of Epidemiology and Preventive Medicine, \\ College of Public Health, National Taiwan University, Taipei City, Taiwan
}

\section{Objective}

This study was to elucidate the spatio-temporal correlations between the mild and severe enterovirus cases through integrating enterovirus-related three surveillance systems in Taiwan. With these fully understanding epidemiological characteristics, hopefully, we can develop better measures and indicators from mild cases to provide early warning signals and thus minimizing subsequent numbers of severe cases.

\section{Introduction}

In July 2012, the 54 children infected with enterovirus-71(EV71) were died in Cambodia [1]. The media called it as mystery illness and made Asian parents worried. In fact, the severe epidemics of enterovirus occurred frequently in Asia, including Malaysia, Singapore, Taiwan and China [2]. The clinical severity varied from asymptomatic to mild (hand-foot-mouth disease and herpangina) and severe pulmonary edema/hemorrhage and encephalitis [3]. Up to now, the development of vaccine for EV-71 and the more effective antiviral drug was still ongoing [4]. Therefore, surveillance for monitoring the enterovirus activity and understanding the epidemiological characteristics between mild and severe enterovirus cases was crucial.

\section{Methods}

Three main databases including national notifiable diseases surveillance, sentinel physician surveillance and laboratory surveillance from July 1, 1999 to December 31, 2008 were analyzed. The Pearson's correlation coefficient was applied for measuring the consistency of the trend. The Poisson space-time scan statistic [5] was used for identifying the most likely clusters. We used GIS (ArcMap, version9.0; ESRI Inc., Redlands, CA, USA) for visualization of detected clusters.

\section{Results}

Temporal analysis found that the Pearson's correlation between mild EV cases and severe EV cases occurring in the same week was $0.553(\mathrm{p}<0.01)$ in Figure 1. Such a correlation became moderate (data) when mild EV cases happened in 1 4 weeks before the current severe EV cases. Among the 1,517 severe EV cases notified to Taiwan CDC during the study period, the mean age was 27 months, $61.4 \%$ was male and $12 \%$ were fatal. These severe EV cases were significantly associated with the positive isolation rate of EV-71, with much higher correlation than the mild cases $[0.498 p<0.01$ vs. 0.278 , $\mathrm{p}<0.01]$. Using the space-time cluster method, we identified three possible clusters in June 2008 in six cities/counties (Figure 2).

\section{Conclusions}

Taiwan's surveillance data indicate that local public health professionals can monitor the trends in the numbers of mild EV cases in community to provide early warning signals for local residents to prevent the severity of future waves.

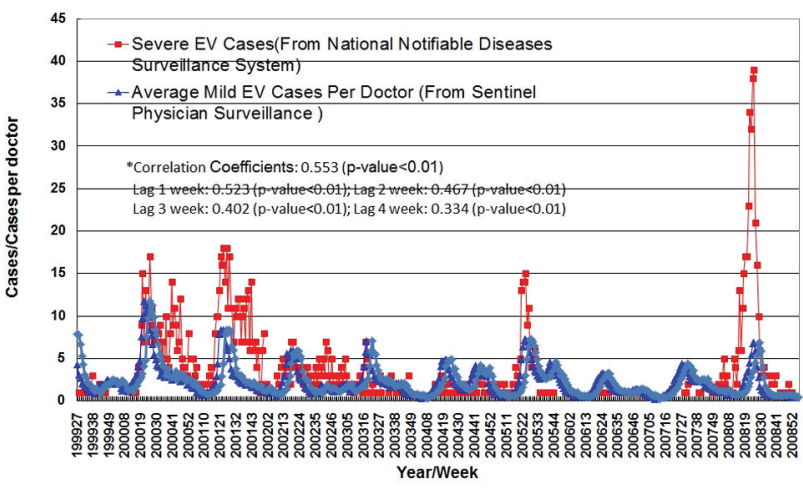

Figure 1. The temporal trend between mild and severe EV cases.

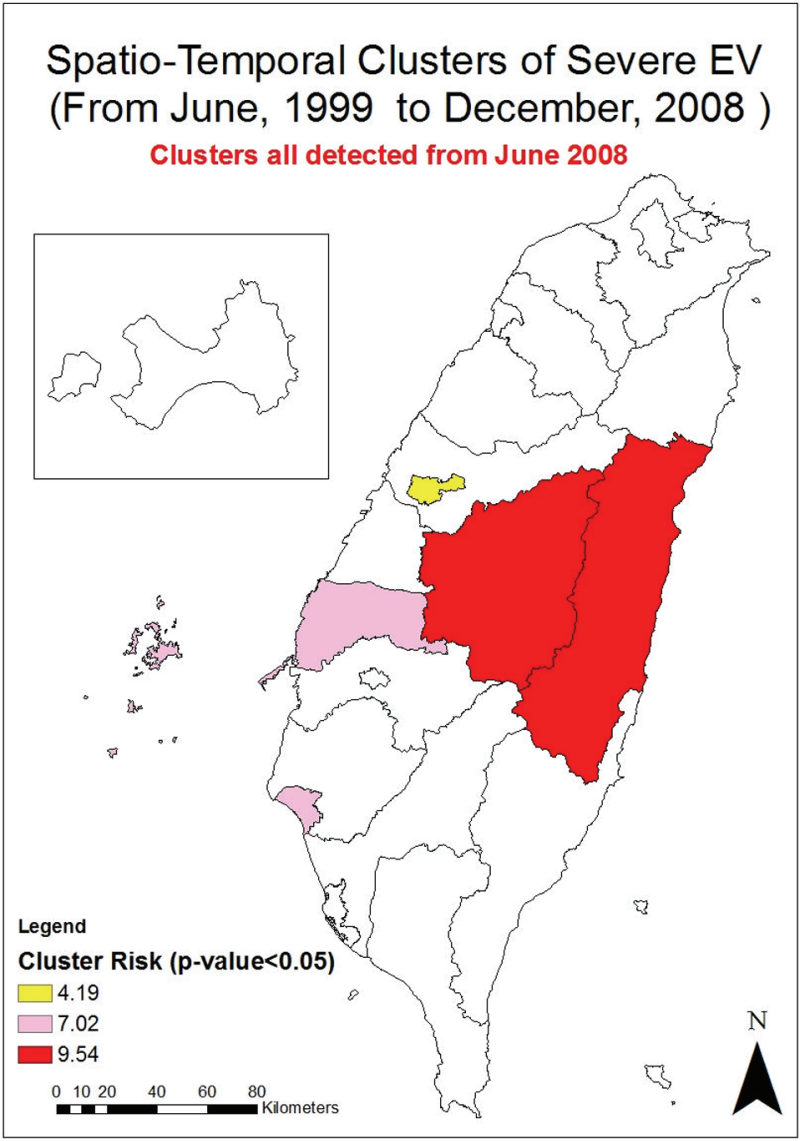

Figure 2. The spatio-temporal clusters of EV severe cases. 


\section{Keywords}

Enterovirus; Suveillance; Space Time Clusters

\section{References}

1. WHO, Severe complications of hand, foot and mouth disease (HFMD) caused by EV-71 in Cambodia - conclusion of the joint investigation[http://www.who.int/csr/don/2012_07_13/en/index.html]

2. Lee MS, Chiang PS, Luo ST, Huang ML, Liou GY, Tsao KC, Lin TY: Incidence rates of enterovirus 71 infections in young children during a nationwide epidemic in Taiwan, 2008-09. PLoS Negl Trop Dis 2012, 6(2):e1476.
3. Chen SC, Chang HL, Yan TR, Cheng YT, Chen KT: An eight-year study of epidemiologic features of enterovirus 71 infection in Taiwan. Am J Trop Med Hyg 2007, 77(1):188-191.

4. Chang LY: Enterovirus 71 in Taiwan. Pediatr Neonatol 2008, 49(4):103-112.

5. Kulldorff M, Athas WF, Feurer EJ, Miller BA, Key CR: Evaluating cluster alarms: a space-time scan statistic and brain cancer in Los Alamos, New Mexico. Am J Public Health 1998, 88(9):1377-1380.

\section{*Ta-Chien Chan}

E-mail: dachianpig@gmail.com 